\title{
BIOLUMINESCENCE IMAGING FOR MEASURING FLUID SHEAR DISTRUBUTIONS
}

\author{
Yasunori Watanabe ${ }^{1}$, Jun Sakai ${ }^{2}$, Yuta Mitobe ${ }^{3}$ and Yasuo Niida ${ }^{4}$ \\ The dinoflagellate Pyrocystis lunula emits light in response to water motion. The statistical \\ features of the bioluminescence, emitted by $P$. lunula, owing to shear stress in oscillatory \\ boundary layer flows over ripped bed were studied in this paper with the aim to develop a \\ new imaging technique for measuring fluid strain rate and shear using plankton that emit light \\ in response to mechanical stimulation. The flash intensity has been found to correlate with \\ fluid strain rate estimated from fluid velocity over ripples. Thus the instantaneous planar \\ distribution of the fluid shear can be estimated from video images of the bioluminescence in \\ a fluid region by using the empirical relation determined in this study.
}

Keywords: Bioluminescence, Shear, Turbulence, Ripple, Image measurement

\section{INTRODUCTION}

In shallow water, turbulence produced in the wave boundary layer disturbs and carries sediments for forming wavy rippled seabed which intensifies fluid shear in the lee of the ripple crest to induce vortices and turbulence. While the shear stress is an important factor to characterize evolution of turbulence and local sediment transport, there is no way to directly measure the stress acting in fluid, whereas it was conventionally estimated from fluid velocity measured in physical experiments.

Some species of marine planktons emit light as a response to mechanical stimulation. Blaser et al. (2002) studied the flash responses of the bioluminescent dinoflagellate Pyrocystis fusiformis in laminar shearing pipe flow. Stokes et al. (2004) applied the bioluminescence imaging technique to ocean breaking waves, where strong turbulence and shears are organized and developed during wave propagation, suggesting a possible application in which a statistical calibration of the cell-flash could be used to measure the actual wave field. Recently, Foti et al. (2010) also studied bioluminescence responses of the dinoflagellate Alexandrium tamarense in the shear field. The authors (2011) have found correlation of the maximum luminescence intensity with the maximum impact water pressure, and statistical features of the flash intensity, which supports potential application of the bioluminescence measurement to quantify the dynamic wave pressure acting on coastal structures. Bioluminescence imaging also has potential advantages for determining planar or volumetric distribution of mechanical stress within a fluid region under ocean waves.

In this paper, correlations of the cell-flash intensity of the dinoflagellate Pyrocystis lunula with the shear strength in oscillatory turbulent boundary layer flows over ripples have been studied with the aim to develop fundamental imaging technique to measure planar shear distributions from video images of the bioluminescence.

\section{EXPERIMENT}

The dinoflagellate planktons Pyrocystis lunula are 20 to $30 \mu \mathrm{m}$ long, and, in response to external stimuli, emit blue light with a dominant wavelength in the range of 470 to $480 \mathrm{~nm}$ (see figure 1). The plankton were cultured in enriched $\mathrm{f} / 2$ media at $20^{\circ} \mathrm{C}$ under cool-white fluorescent lighting with a $12 \mathrm{~h}$ light / $12 \mathrm{~h}$ dark cycle.

Seawater mixed with $P$. lunula was placed in a U-tube type of transparent oscillatory flow channel with a ripple-shaped bottom (See figure 2). Sinusoidal oscillatory flows with wave period of $1.08 \mathrm{~s}$ and amplitude of $20 \mathrm{~cm}$ were generated in the channel. A 12-bit high-sensitivity digital video camera with an acquisition frequency of $40 \mathrm{~Hz}$ was placed to record the planar distribution of cells over the ripple from a side wall of the channel.

\footnotetext{
${ }^{1}$ School of Engineering, Hokkaido University, North 13 West 8, Sapporo 060 8628, Japan

2 Docon co. Itd, Atsubetsu-chuo, 1-5-4-1Sapporo 004 8585, Japan

${ }^{3}$ School of Engineering, Hokkaido University, North 13 West 8, Sapporo 060 8628, Japan

${ }^{4}$ School of Engineering, Hokkaido University, North 13 West 8, Sapporo 060 8628, Japan
} 

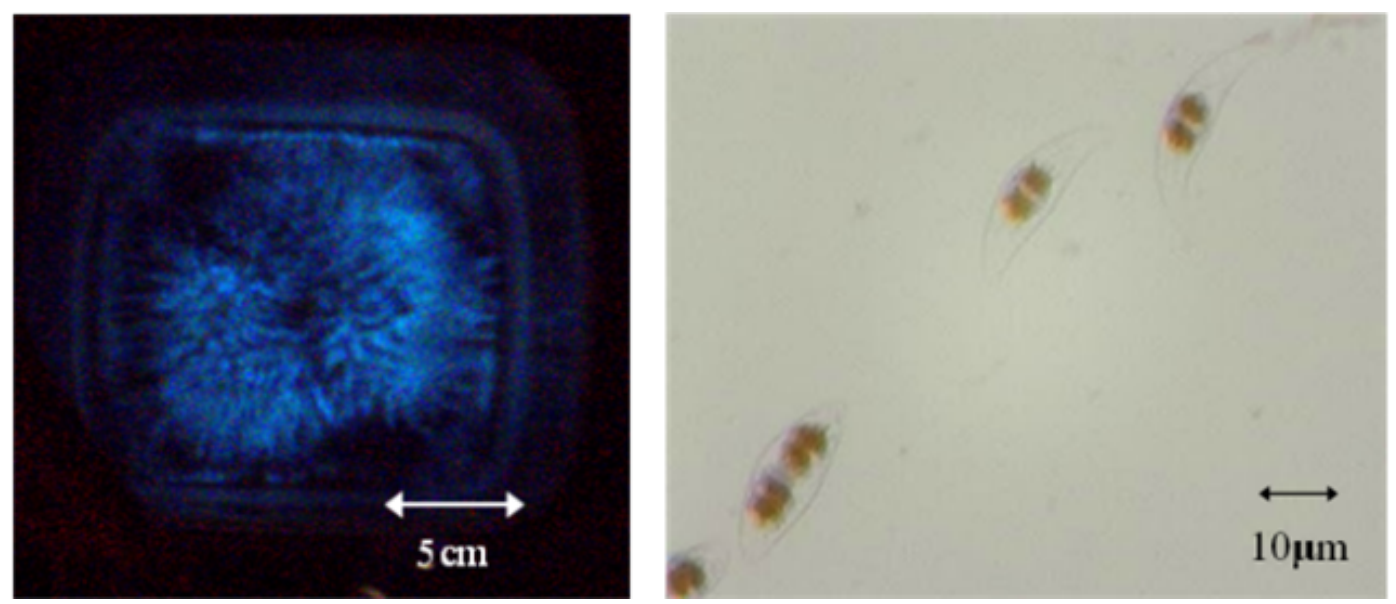

Figure 1. Flashing Pyrocystis lunula (left) and microscope photograph of P. lunula (right).

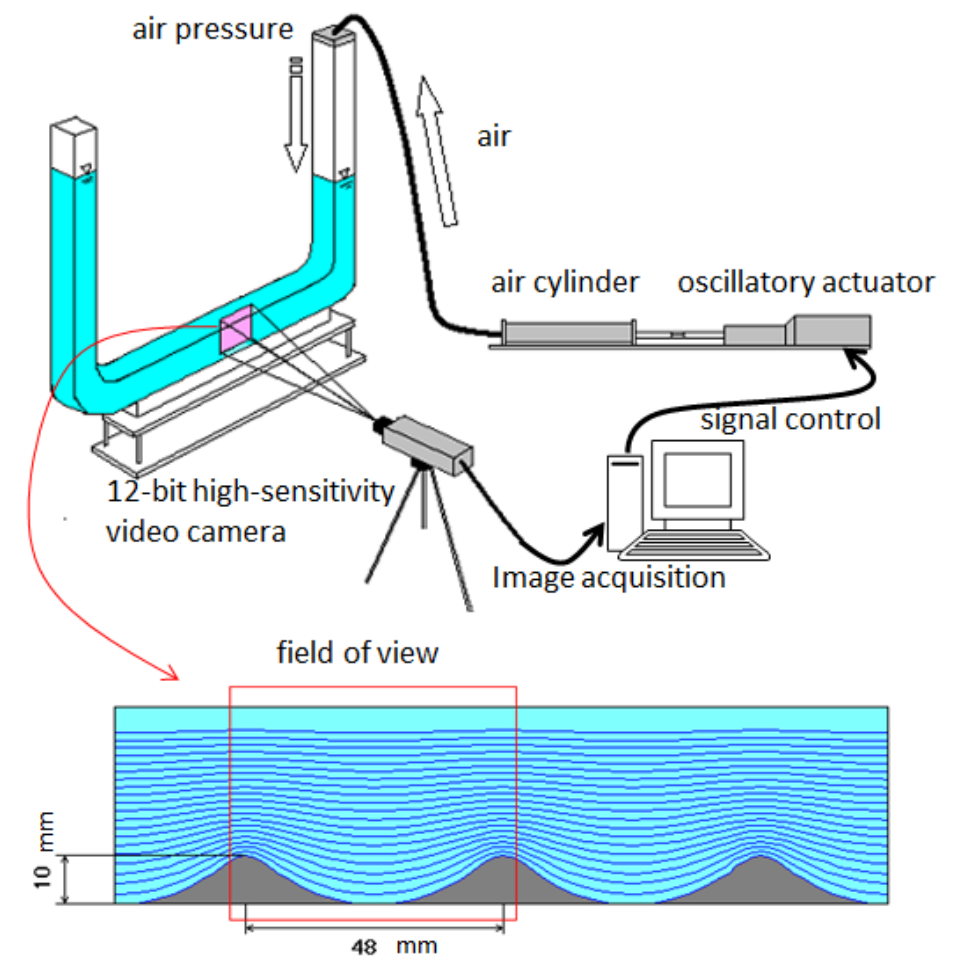

Figure 2. Experimental setup and ripple-shaped bottom installed in the channel.

The acquired image intensity was calibrated with the optical intensity of the bioluminescence. A LED array panel emitting continuous blue light with the peak wavelength of $474 \mathrm{~nm}$, which was identical to the dinoflagellate luminescence, was recorded with the camera (see figure 3 ). The photoconductivity power for the light intensities was measured using a photodiode for calibrating the image intensity of the corresponding light. In this calibration procedure, the cell-flash intensity 

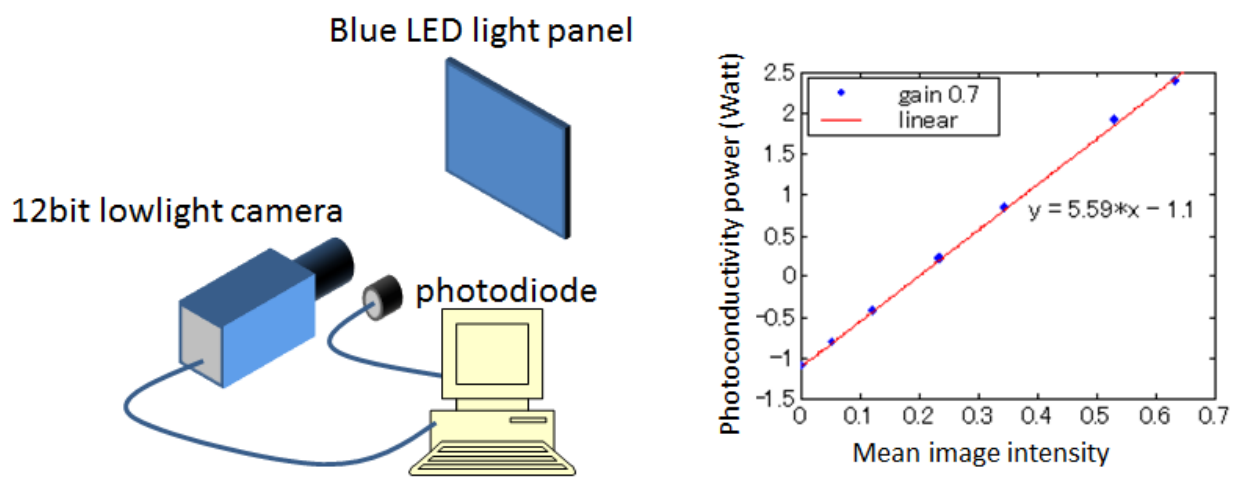

Figure 3. Experimental setup to calibrate the image intensity with photo-conductivity measured by a photo-diode (left) and example of a linear relation of the image intensity and photo-conductivity power (right).
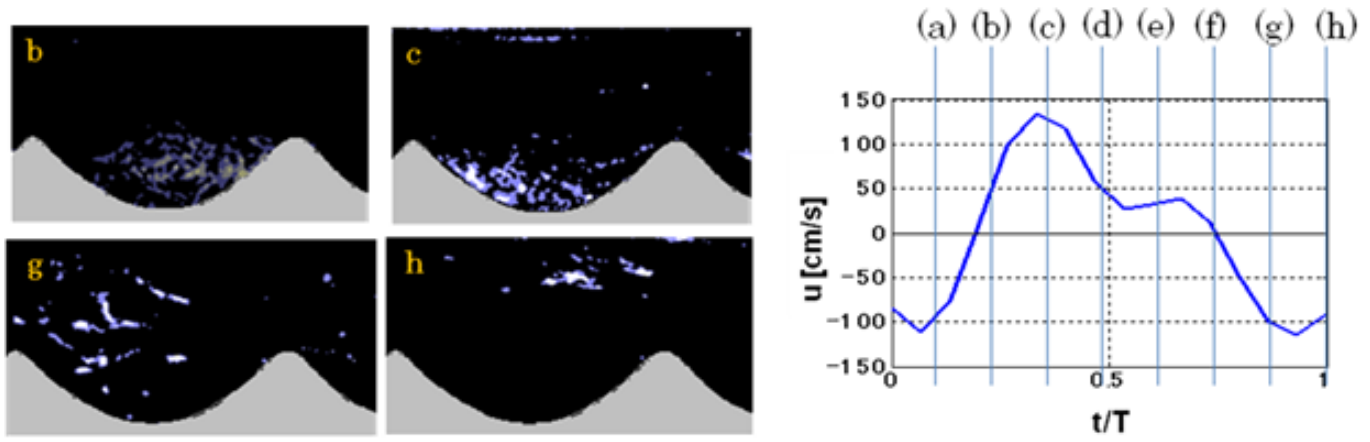

Figure 4. Typical cell-flash distributions over the ripple (left) and time record of the spatial-averaged horizontal velocity over the ripple (right).

was expressed as a measure of the photoconductivity power (Watt).

Keane et al. (1995) developed super-resolution particle imaging velocimetry (SRPIV), which is based on a combination of cross-correlation and tracking methods, in order to enhance the resolution of PIV and to measure small-scale turbulence. The planar fluid velocity over the ripple was also measured by using SRPIV for seeking flow parameters to trigger the cell-flash. In the PIV measurements, neutral buoyant particles with diameter of about $50 \mu \mathrm{m}$ are mixed in water to be illuminated by a YAG laser sheet installed. The displacement of the particles was recorded by a high-speed video camera at the recording frequency of $250 \mathrm{~Hz}$. The ensemble flash distributions and fluid flow velocity at eight successive phases over one wave period were statistically examined through 20 trials of experiments.

The biological behavior of dinoflagellates depends on a circadian biological rhythm. The details of experimental conditions to control flash activities can be found in Watanabe and Tanaka (2010).

\section{RESULTS}

The typical flash behaviors were observed according to separation of the boundary layer, and ejection of vortices from the crest in the oscillatory boundary layer flow over ripples (see figure 4).

Figure 5 shows the distributions of the absolute strain rate, $|\partial u / \partial z+\partial w / \partial x|$, where $u$ and $w$ are the ensemble velocity. It is known that a finite difference representation of discrete PIV results for velocity gradients may cause a significant error since the measurement error is amplified in the 

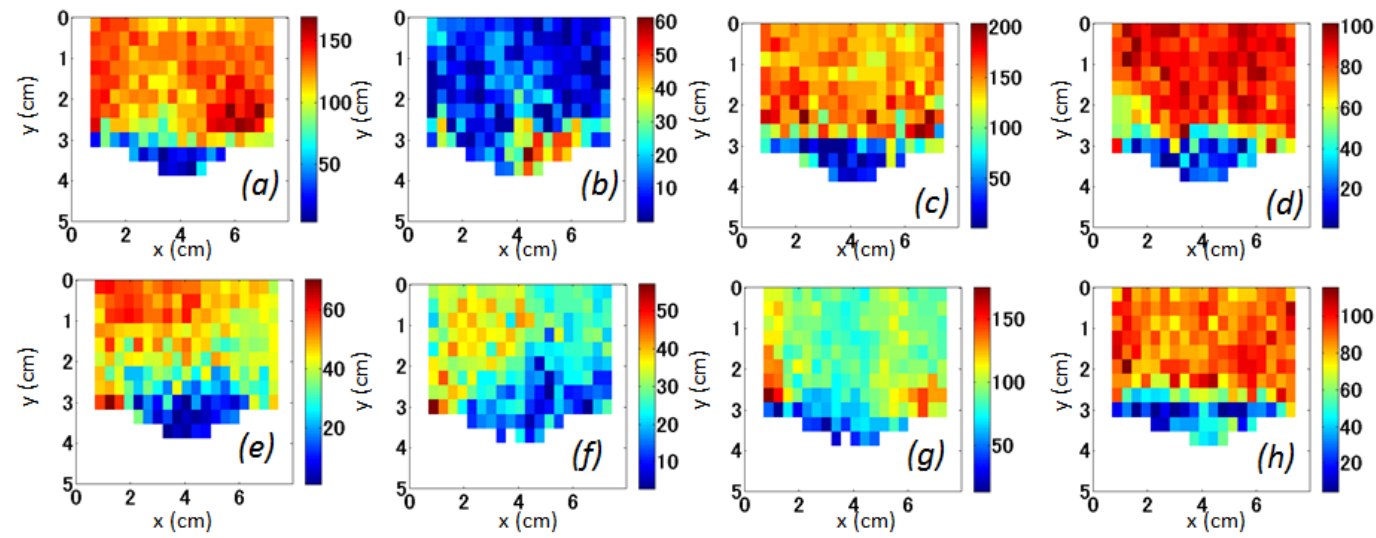

Figure 5. Distributions of the absolute strain rates during one wave period (phase (a) to (h) are indicated in figure 4). Unit: $s^{-1}$
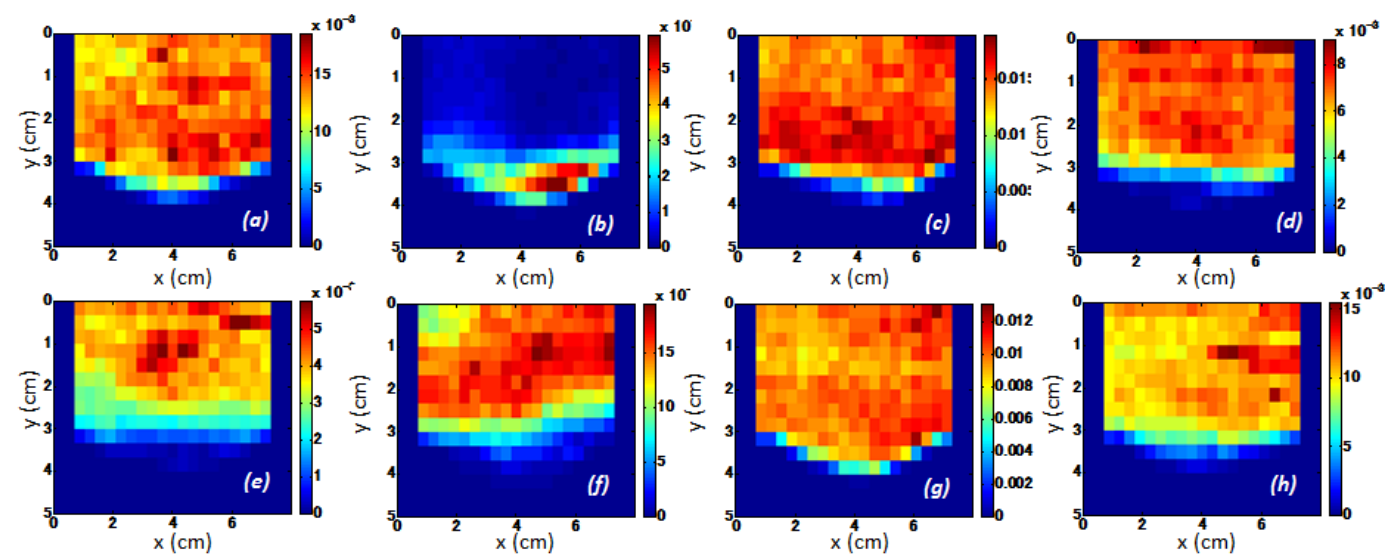

Figure 6. Distributions of the flash intensity during one wave period (phase (a) to (h) are indicated in figure 4). Unit: Watt

differencing procedure. In this study, a MLS scheme proposed by Takehara \& Eto (2010) was used for estimating reasonable distributions of the strain rate; that is, the particle velocities estimated by Super-resolution PIV are interpolated over dispersed particles by polynomials to obtain higher order approximation of the velocity gradients. It is observed from figure 5 that high strain rate is developed above the crest level at the phase (a), (c) and (d), indicating a typical shear layer formed over the ripple, while the local strain concentrates in a separation vortex produced behind the crest at the phase (b).

The ensemble averages of the normalized flash intensities at each phase shows the flash intensities also distribute in the corresponding high strain regions (figure 6), indicating that the dinoflagellate flashes as a response of the fluid shear stress that is proportional to the strain.

The time series of the flash intensity and the measured absolute shear averaged over one ripple are compared in figure 7. Identical features of the temporal variations between them indicate possible estimations of the fluid shear by using the bio-luminescence imaging.

It is found that there is a linear correlation between the normalized strain and cell-intensity at all phases during one wave period (see figure 8). The correlation coefficient was estimated to be 0.92 . This correlation provides the empirical relation to approximate the absolute fluid shear $(\tau)$ from the 

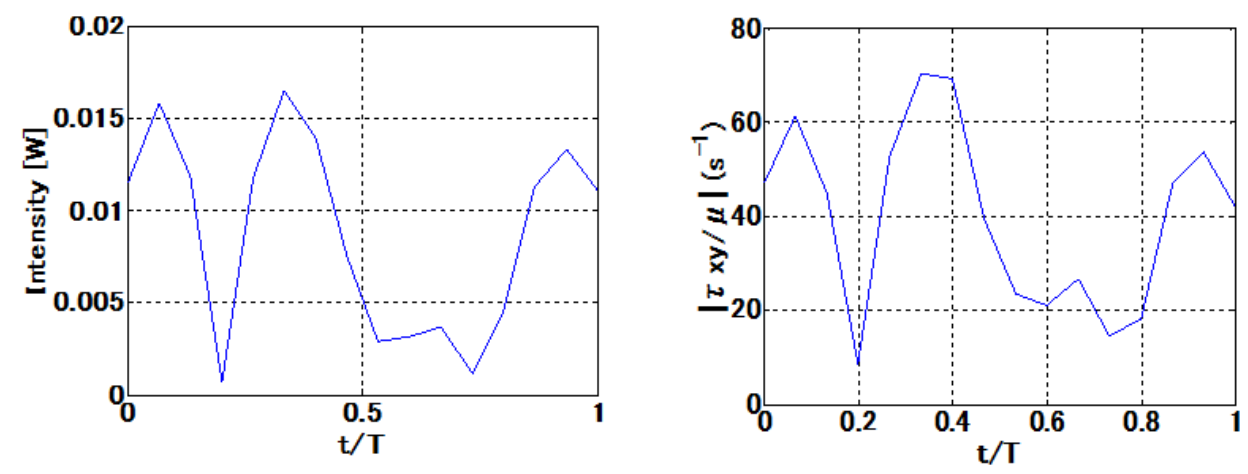

Figure 7. Time records of the spatial-averaged flash intensity (left) and absolute strain (right).

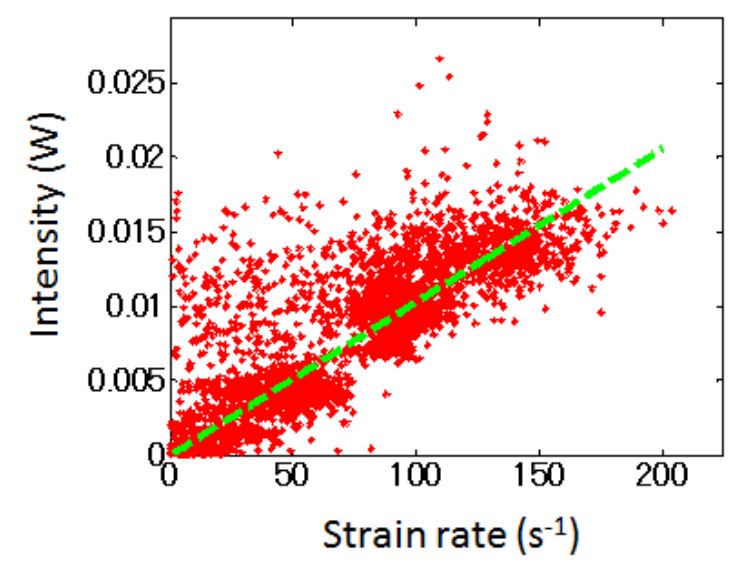

Figure 8. Scatter plots of the flash intensity for the absolute strain over all corresponding locations during one wave period.

flash intensity $(I)$ :

$$
\tau=9.712 \times 10^{3} \mu I,
$$

where $\mu$ is the viscosity. We can directly estimate simultaneous shear within a fluid region from bioluminescence images using the above relation. It should be noted that the dispersions appearing in figure 8 may be influenced by biological factors such as fatigue of dinoflagellate, response memory and flash duration, which needs to be investigated for the practical application of the bioluminescence technique to measure the fluid stress.

It is also interesting to investigate contributions of turbulent stimulations to the cell-flash. Figure 9 shows the spatial distributions of the turbulent kinetic energy at each phase. We found from the comparisons with figure 6 that there is no correlation of the flash intensity with the turbulence kinetic energy developing in the boundary layer, indicating the bioluminescence of P.lunula reflect only the instantaneous shear even in turbulent flows.

\section{CONCLUSIONS}

A relation between bioluminescence of dinoflagellate Pyrocystis lunula and fluid shear in oscillatory turbulent flows over a ripple was investigated in this paper. The flash intensity was found to highly correlate with fluid strain rate estimated from the fluid velocity measured by using 

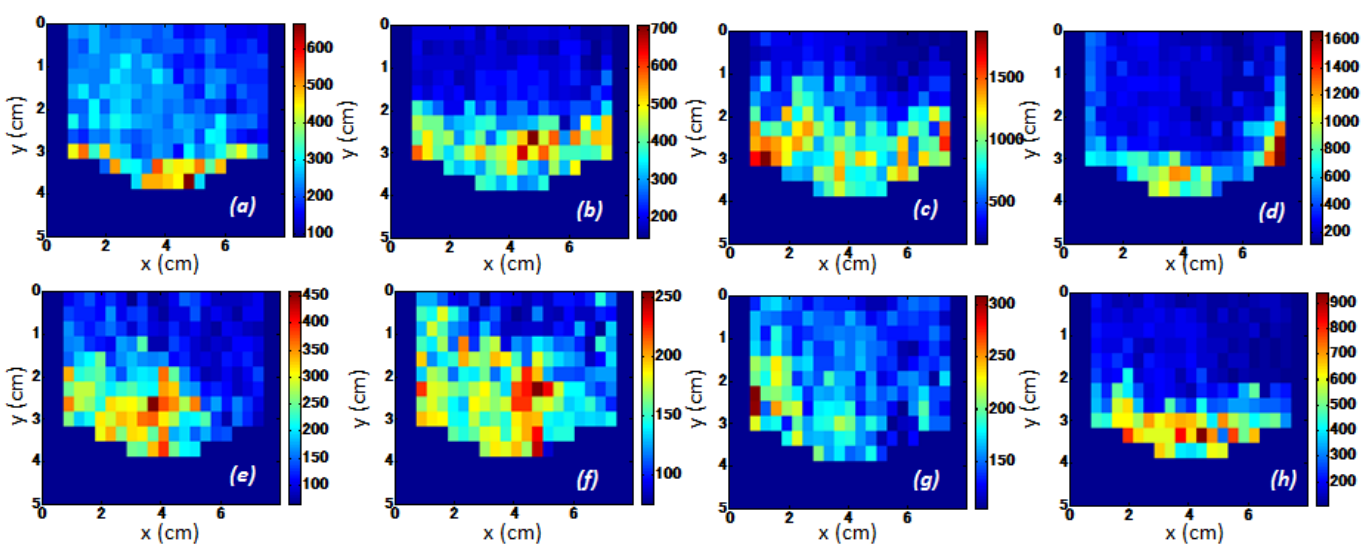

Figure 9. Distributions of the turbulent kinetic energy during one wave period (phase (a) to (h) are indicated in figure 4). Unit: $m m^{2} s^{-2}$

super-resolution Particle Imaging Velocimetry. The instantaneous planar distribution of the fluid shear can be estimated from video images of the bioluminescence in a fluid region by using the empirical relation determined in this study.

Further examinations of the measurement accuracies and biological uncertainties should be investigated for practical applications of the bioluminescence technique.

\section{ACKNOWLEDGMENTS}

Financial support for this study is provided by JSPS Grants-in-Aid Scientific Research (21656120).

\section{REFERENCES}

Blaser S., Kurisu F., Satoh H., Mino T., Hydromechanical stimulation of bioluminescent plankton, Luminescence, 17, 370 - 380 (2002)

Foti E., Faraci C., Foti R., Bonanno G., On the use of bioluminescence for estimating shear stresses over a rippled seabed, Meccanica, 45, $881-895$ (2010)

Stokes M.D., Deane G.B., Latz M.I., Rohr J., Bioluminescence imaging of wave-induced turbulence, J. Geophys. Res., 109, C01004, doi:10.1029/2003JC001871 (2004)

Takehara K., Etoh T., A proposal of a vorticity estimation method with MLS and PTV, and its apprication to flows under wind waves, Doboku Gakkai Ronbunshuu B, 65 (3), 151-165 (2009)

Watanabe Y. and Tanaka Y., Bioliminescence-based imaging technique for pressure measurement in water, Experiments in Fluids, 51, 225 - 236 (20011) 